\title{
Some Notions of Separability of Metric Spaces in ZF and Their Relation to Compactness
}

by

\author{
Kyriakos KEREMEDIS \\ Presented by Czestaw BESSAGA
}

Summary. In the realm of metric spaces we show in $\mathbf{Z F}$ that:

(1) Quasi separability (a metric space $\mathbf{X}=(X, d)$ is quasi separable iff $\mathbf{X}$ has a dense subset which is expressible as a countable union of finite sets) is the weakest property under which a limit point compact metric space is compact.

(2) $\omega$-quasi separability (a metric space $\mathbf{X}=(X, d)$ is $\omega$-quasi separable iff $\mathbf{X}$ has a dense subset which is expressible as a countable union of countable sets) is a property under which a countably compact metric space is compact.

(3) The statement "Every totally bounded metric space is separable" does not imply the countable choice axiom CAC.

1. Notation and terminology. Let $\mathbf{X}=(X, d)$ be a metric space, $x \in X$ and $\varepsilon>0$. Then $B(x, \varepsilon)=\{y \in X: d(x, y)<\varepsilon\}$ denotes the open ball in $\mathbf{X}$ with center $x$ and radius $\varepsilon$. Given $B \subseteq X, \delta(B)=\sup \{d(x, y)$ : $x, y \in B\} \in \mathbb{R}_{+} \cup\{\infty\}$ will denote the diameter of $B$.

$\mathbf{X}$ is said to be bounded iff $\delta(X)<\infty$.

$\mathbf{X}$ is said to be separable iff $\bar{D}=X$ for some countable subset $D$ of $X$.

Let $\mathcal{U}$ be an open cover of $\mathbf{X}$. We say that $\mathcal{U}$ has a Lebesgue number $\delta>0$ iff for every $A \subseteq X$ with $\delta(A)<\delta$ there exists $U \in \mathcal{U}$ with $A \subseteq U$.

Given $\varepsilon>0$, a subset $D$ of $X$ is called $\varepsilon$-dense iff for every $x \in X$, $B(x, \varepsilon) \cap D \neq \emptyset$, or equivalently $X=\bigcup\{B(d, \varepsilon): d \in D\}$. A finite $\varepsilon$-dense set $D$ of $\mathbf{X}$ is called an $\varepsilon$-net.

2010 Mathematics Subject Classification: 03E25, 54D65, 54D30.

Key words and phrases: axiom of choice, separable, compact, countably compact, sequentially compact, complete, totally bounded and Lebesgue metric spaces.

Received 9 August 2016; revised 6 December 2016.

Published online 20 December 2016. 
$\mathbf{X}$ is said to be Lebesgue iff every open cover of $\mathbf{X}$ has a Lebesgue number.

$\mathbf{X}$ is said to be almost separable iff for every $\varepsilon>0$ there is a countable (finite or countably infinite) $\varepsilon$-dense subset $D$ of $X$.

$\mathbf{X}$ is said to be strongly almost separable iff it admits a sequence $\left(D_{n}\right)_{n \in \mathbb{N}}$ such that for all $n \in \mathbb{N}, D_{n}$ is a countable $1 / n$-dense subset $\mathbf{X}$.

$\mathbf{X}$ is said to be totally bounded iff for every $\varepsilon>0$, there exists an $\varepsilon$-net of $\mathbf{X}$.

$\mathbf{X}$ is said to be strongly totally bounded iff it admits a sequence $\left(D_{n}\right)_{n \in \mathbb{N}}$ such that for all $n \in \mathbb{N}, D_{n}$ is a $1 / n$-net of $\mathbf{X}$.

$\mathbf{X}$ is said to be precompact iff for every $\varepsilon>0, X$ can be covered by finitely many open discs of radius $\varepsilon$.

$\mathbf{X}$ is said to be strongly precompact iff it admits a sequence $\left(\mathcal{U}_{n}\right)_{n \in \mathbb{N}}$ such that for every $n \in \mathbb{N}, \mathcal{U}_{n}$ is a finite cover of $\mathbf{X}$ consisting of open discs of radius $1 / n$.

$\mathbf{X}$ is said to be compact or Heine-Borel compact iff each of its open covers has a finite subcover.

$\mathbf{X}$ is said to be countably compact iff each of its countable open covers has a finite subcover.

$\mathbf{X}$ is said to be sequentially compact iff each of its sequences has a limit point.

$\mathbf{X}$ is said to be limit point compact iff every infinite subset of $X$ has a limit point.

$\mathbf{X}$ is said to be sequentially bounded iff each of its sequences admits a Cauchy subsequence.

$\mathbf{X}$ is said to be complete iff each of its Cauchy sequences converges.

$\mathbf{X}$ is Cantor complete iff $\bigcap\left\{G_{n}: n \in \omega\right\} \neq \emptyset$ for every descending family $\left\{G_{n}: n \in \omega\right\}$ of non-empty closed subsets of $\mathbf{X}$ with $\lim _{n \rightarrow \infty} \delta\left(G_{n}\right)=0$.

We recall that if $\left\{\mathbf{X}_{n}=\left(X_{n}, d_{n}\right): n \in \mathbb{N}\right\}$ is a family of metric spaces then the function $d: X \times X \rightarrow \mathbb{R}, X=\prod_{n \in \mathbb{N}} X_{n}$, given by

$$
d(x, y)=\sum_{n \in \mathbb{N}} \frac{\rho_{n}\left(x_{n}, y_{n}\right)}{2^{n}},
$$

where $\rho_{n}(a, b)=\min \left\{1, d_{n}(a, b)\right\}$ for all $n \in \mathbb{N}$, is a metric, and the metric topology it induces coincides with the product topology. We shall always assume that whenever a family $\left\{\mathbf{X}_{n}=\left(X_{n}, d_{n}\right): n \in \mathbb{N}\right\}$ of metric spaces is given, then $\delta\left(X_{n}\right) \leq 1$ for all $n \in \mathbb{N}$, and the product $X=\prod_{n \in \mathbb{N}} X_{n}$ carries the metric $d$.

An infinite set $X$ is said to be Dedekind-infinite (resp. weakly Dedekindinfinite) iff $X$ contains a countably infinite set (resp. $\mathcal{P}(X)$ contains a countably infinite set). Otherwise $X$ is said to be Dedekind-finite (resp. weakly Dedekind-finite). 
Below we list some of the weak forms of the axiom of choice we shall deal with.

- CAC (Form 8 of [4]): For every countable family $\mathcal{A}$ of non-empty sets there exists a function $f$ such that $f(x) \in x$ for all $x \in \mathcal{A}$.

- $\mathbf{C A C}(\mathbb{R})($ Form 94 of [4]): CAC restricted to families of non-empty subsets of $\mathbb{R}$. Equivalently (see e.g. [2]), every family $\mathcal{A}=\left\{A_{n}: n \in \mathbb{N}\right\}$ of disjoint dense subsets of $\mathbb{R}$ has a choice set.

- $\mathbf{C A C}_{\omega}(\mathbb{R})$ (Form 5 of $[4]$ ): $\mathbf{C A C}(\mathbb{R})$ restricted to families of nonempty countable subsets of $\mathbb{R}$. Equivalently (see e.g. [3]), every countable family of disjoint non-empty countable subsets of the real line has an infinite subfamily with a choice function.

- CUC (Form 31 of [4]): The countable union of countable sets is countable.

- $\mathbf{C A C}_{\text {fin }}$ (Form 10 of [4]): CAC restricted to countable families of nonempty finite sets. Equivalently (see Form [10 O] in [4]), every infinite well-ordered family $\mathcal{A}$ of non-empty finite sets has a partial choice set, i.e., some infinite subfamily $\mathcal{B}$ of $\mathcal{A}$ has a choice set.

- IWDI (Form 82 of [4]): Every infinite set is weakly Dedekind infinite.

2. Introduction and some preliminaries and known results. In this paper, the intended context for reasoning and statements of theorems will be the Zermelo-Fraenkel set theory $\mathbf{Z F}$. To indicate that a result is proved in $\mathbf{Z F}$ (resp. ZFC (= ZF $+\mathbf{A C})$ ) we shall start the statements of the theorems and propositions with $(\mathbf{Z F})$ (resp. (ZFC)).

The notions of strongly totally bounded (stb for abbreviation), strongly precompact (sp), quasi separable (qs), $\omega$-quasi separable ( $\omega$-qs), almost separable (as) and strongly almost separable (sas) are new here. Some of these notions are consequences of separability but some others are not. However, in $\mathbf{Z F}+\mathbf{C A C}$ they all imply separability. The following $\mathbf{Z F}$ implications:

- $\mathbf{s}$ (separable) $\rightarrow p, \forall p \in\{\mathbf{q s}, \omega$-qs, as, $\mathbf{s a s}\}$,

- $\mathbf{q s} \rightarrow \omega$-qs, sas $\rightarrow p, \forall p \in\{\omega$-qs, as $\}$,

- tb (totally bounded) $\leftrightarrow \mathbf{p}$ (precompact),

- $\mathbf{s p} \rightarrow \mathbf{p}$,

- $\mathbf{t b} \rightarrow \mathbf{a s}$, and

- $\mathbf{s t b} \rightarrow p, \forall p \in\{\mathbf{t b}, \mathbf{a s}, \mathbf{s a s}, \boldsymbol{\omega}$-qs, $\mathbf{s p}\}$

are straightforward and are left as a warm-up exercise for the reader. We also observe:

1. The real line $\mathbb{R}$ has each of the properties listed in $A=\{\mathbf{s}, \mathbf{a s}, \mathbf{s a s}$, $\mathbf{q s}, \omega$-qs $\}$ but none of the properties listed in $B=\{\mathbf{t b}, \mathbf{s t b}, \mathbf{s p}\}$. Hence, the non-implications $p \nrightarrow q, p \in A, q \in B$, hold true in $\mathbf{Z F}$. 
2. In the basic Cohen model $\mathcal{M} 1$ in [4], the subspace $\mathbf{X}, X=A \cap(0,1)$, of the real line $\mathbb{R}$, where $A$ denotes the set of all added Cohen reals, is totally bounded but has none of the properties listed in $C=\{\mathbf{s}, \mathbf{s a s}, \mathbf{q s}, \omega-\mathbf{q s}, \mathbf{s t b}, \mathbf{s p}\}$ (recall that $A$ is a dense, Dedekind finite subset of $\mathbb{R}$ ). Hence, all non-implications tb $\nrightarrow p, p \in C$, are consistent with $\mathbf{Z F}$.

Table 1 below contains the $\mathbf{Z F}$ implications/non-implications which we shall establish in this article, as well as the $\mathbf{Z F}$ implications/non-implications which remain open. The interpretation of the table is self-evident: If the entry with row heading $x$ and column heading $y$ is $\rightarrow$ (resp. $\rightarrow$ ) then $x \rightarrow y$ (resp. $x \nrightarrow y$ ). If it is ? then it is not known whether $x \rightarrow y$ or $x \nrightarrow y$.

Table 1

\begin{tabular}{|c|c|c|c|c|c|c|c|c|}
\hline & stb & sp & $\mathrm{s}$ & qs & $\omega$-qs & tb & sas & as \\
\hline stb & $\rightarrow$ & $\rightarrow$ & $\nrightarrow$ & $\rightarrow$ & $\rightarrow$ & $\rightarrow$ & $\rightarrow$ & $\rightarrow$ \\
\hline sp & ? & $\rightarrow$ & $\nrightarrow$ & ? & ? & $\rightarrow$ & ? & $?$ \\
\hline s & $\nrightarrow$ & $\nrightarrow$ & $\rightarrow$ & $\rightarrow$ & $\rightarrow$ & $\nrightarrow$ & $\rightarrow$ & $\rightarrow$ \\
\hline qs & $\nrightarrow$ & $\nrightarrow$ & $\nrightarrow$ & $\rightarrow$ & $\rightarrow$ & $\nrightarrow$ & $\nrightarrow$ & $\nrightarrow$ \\
\hline$\omega$-qs & $\nrightarrow$ & $\nrightarrow$ & $\nrightarrow$ & $\nrightarrow$ & $\rightarrow$ & $\nrightarrow$ & $\nrightarrow$ & $\nrightarrow$ \\
\hline tb & $\nrightarrow$ & $\nrightarrow$ & $\nrightarrow$ & $\nrightarrow$ & $\nrightarrow$ & $\rightarrow$ & $\nrightarrow$ & $\rightarrow$ \\
\hline sas & $\nrightarrow$ & $\nrightarrow$ & $\nrightarrow$ & $\nrightarrow$ & $\rightarrow$ & $\nrightarrow$ & $\rightarrow$ & $\rightarrow$ \\
\hline as & $\nrightarrow$ & $\nrightarrow$ & $\nrightarrow$ & $\nrightarrow$ & $\nrightarrow$ & $\nrightarrow$ & $\nrightarrow$ & $\rightarrow$ \\
\hline
\end{tabular}

The following has been established in [9], [6] and [7]:

Theorem 1. Let $\mathbf{X}=(X, d)$ be a metric space. Then:

(a) [6] (ZFC) The following are equivalent:

(i) $\mathbf{X}$ is compact.

(ii) $\mathbf{X}$ is limit point compact.

(iii) $\mathbf{X}$ is sequentially compact.

(iv) $\mathbf{X}$ is Cantor complete and totally bounded.

(v) $\mathbf{X}$ is complete and totally bounded.

(vi) $\mathbf{X}$ is complete and sequentially bounded.

(vii) $\mathbf{X}$ is countably compact.

(b) 6] (ZF) If $\mathbf{X}$ is separable then (i)-(vii) are equivalent.

(c) 9] (ZF) $\mathbf{X}$ is sequentially compact iff it is complete and sequentially bounded.

(d) [9] (ZF) $\mathbf{X}$ is compact iff it is totally bounded and Lebesgue.

(e) [7 ( $\mathbf{Z F ) ~ E v e r y ~ s e q u e n t i a l l y ~ c o m p a c t ~ m e t r i c ~ s p a c e ~ i s ~ c o m p a c t ~ i f f ~ e v e r y ~}$ compact metric space is separable. 
Table 2 records the implications/non-implications which hold between the forms of compactness listed in Theorem 1. In Table 2, c, lpc, sc, ctb, cctb and cc abbreviate: compact, limit point compact, sequentially compact, complete and totally bounded, Cantor complete and totally bounded and countably compact respectively.

Table 2

\begin{tabular}{lllllll|}
\hline & c & lpc & sc & ctb & cctb & cc \\
c & $\rightarrow$ & $\rightarrow$ & $\rightarrow$ & $\rightarrow$ & $\rightarrow$ & $\rightarrow$ \\
lpc & $\nrightarrow$ & $\rightarrow$ & $\rightarrow$ & $\nrightarrow$ & $\nrightarrow$ & $\nrightarrow$ \\
sc & $\nrightarrow$ & $\nrightarrow$ & $\rightarrow$ & $\nrightarrow$ & $\nrightarrow$ & $\nrightarrow$ \\
ctb & $\nrightarrow$ & $\nrightarrow$ & $\rightarrow$ & $\rightarrow$ & $\nrightarrow$ & $\nrightarrow$ \\
cctb & $\nrightarrow$ & $\nrightarrow$ & $\rightarrow$ & $\rightarrow$ & $\rightarrow$ & $\nrightarrow$ \\
cc & $\nrightarrow$ & $\nrightarrow$ & $\rightarrow$ & $\nrightarrow$ & $\nrightarrow$ & $\rightarrow$ \\
\hline
\end{tabular}

For the non-implications of Table 2 counterexamples are provided in [6]. Since the c-line and the sc-column of Table 2 contain only $\rightarrow$ 's, it follows that Heine-Borel compactness is the strongest and sequential compactness is the weakest form respectively among all forms of compactness listed in Theorem 1. Also, part (b) of Theorem 1 indicates that in $\mathbf{Z F}$, compactness $=$ sequential compactness in the class $\mathcal{S}$ of all separable metric spaces. In view of the latter observation one may ask:

- Is there a property $p$ weaker than separability such that the statement $\mathbf{s c} \rightarrow \mathbf{c}$ ("Every sequentially compact metric space is compact") (resp. $\mathbf{c t b} \rightarrow \mathbf{c}, \mathbf{c c t b} \rightarrow \mathbf{c}, \mathbf{l p c} \rightarrow \mathbf{c}, \mathbf{c c} \rightarrow \mathbf{c})$ is true in $\mathbf{Z F} ?$

REMARK 1. In view of the following observations, $p$ cannot be second countability.

(1) In $\mathcal{M} 1$ the subspace $\mathbf{A}$ of the real line $\mathbb{R}$ is second countable and sequentially compact. Indeed, every sequence in $\mathbf{A}$ has a finite range. Therefore, every sequence has a limit point and $\mathbf{A}$ is sequentially compact. However, $\mathbf{A}$ is not compact because the open cover $\mathcal{U}=\{(n, n+1) \cap A: n \in \mathbb{Z}\}$ of $\mathbf{A}$ has no finite subcover.

(2) The subspace $\mathbf{A}$ of the real line $\mathbb{R}$ mentioned in (1) is also limit point compact. Indeed, if $K \subseteq A$ is an infinite subset of $A$ without limit points then $\mathbf{K}$ is a second countable, discrete metric space. Therefore, if $\mathcal{B}$ is a countable base for $\mathbf{K}$ then $\{\{x\}: x \in K\} \subseteq \mathcal{B}$, and consequently $K$ is a countably infinite subset of $A$, contradicting the fact that $A$ is Dedekind finite.

(3) The subspace $\mathbf{X}$ of $\mathbb{R}$ mentioned in item 2 at the beginning of the section is second countable, complete (every Cauchy sequence in $\mathbf{X}$ has a finite range), limit point compact (for the same reason as $\mathbf{A}$ in (2)) and totally bounded but not compact. 
We list the following theorems for future reference.

THEOREM 2.

(a) [6] (ZF) The product $\mathbf{X}=\prod_{i \in \omega} \mathbf{X}_{i}$ of a family $\left\{\left(X_{i}, d_{i}\right): i \in\right.$ $\omega\}$ of totally bounded (resp. sequentially compact, complete, Cantor complete) metric spaces is totally bounded (resp. sequentially compact, complete, Cantor complete).

(b) [6] $(\mathbf{Z F})$ Let $\mathbf{X}=(X, d)$ be a metric space and $D$ be a dense subset of $\mathbf{X}$. Then:

(i) $\mathbf{X}$ is totally bounded iff $\mathbf{D}$ is totally bounded.

(ii) $\mathbf{X}$ is Lebesgue iff $\mathbf{D}$ is Lebesgue.

TheOREm 3 ([7]). The statement "Every sequentially compact metric space is compact" implies each of the following:

(i) The product of a countable family of compact metric spaces is compact.

(ii) Every family $\mathcal{A}=\left\{A_{i}: i \in \omega\right\}$ of non-empty compact metric spaces has a choice function.

(iii) Every sequentially compact metric space is separable.

Next we observe that, in contrast to Theorem 2(a), countable compactness is not countably productive in $\mathbf{Z F}$.

THEOREM 4.

(i) The statement "The countable product of countably compact metric spaces is countably compact" implies "Every countable family of

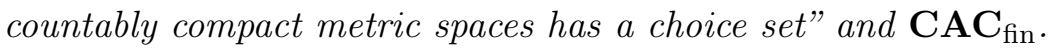

(ii) The statement "Every countably compact metric space is compact" implies IWDI.

Proof. (i) Fix a family $\left\{\mathbf{X}_{n}=\left(X_{n}, d_{n}\right): n \in \mathbb{N}\right\}$ of countably compact metric spaces. Without loss of generality we may assume that each member of the family has diameter $\leq 1$. For every $n \in \mathbb{N}$, let $\infty_{n} \notin X_{n}, Y_{n}=X_{n} \cup\left\{\infty_{n}\right\}$ and define a metric $\rho_{n}: Y_{n} \times Y_{n} \rightarrow \mathbb{R}$ by

$$
\rho_{n}(x, y)= \begin{cases}0 & \text { if } x=y, \\ d_{n}(x, y) & \text { if } x, y \in X_{n}, \\ 1 & \text { if } x \in X_{n} \text { and } y=\infty_{n} .\end{cases}
$$

Clearly, for every $n \in \mathbb{N}, \mathbf{Y}_{n}$ is a countably compact metric space. By our hypothesis, $\mathbf{Y}=\prod_{n \in \mathbb{N}} \mathbf{Y}_{n}$ is countably compact. Since $\mathcal{G}=\left\{\pi_{n}^{-1}\left(X_{n}\right)\right.$ : $n \in \mathbb{N}\}$ is clearly a countable family of closed subsets of $\mathbf{X}$ with the finite intersection property, it follows that $\bigcap \mathcal{G} \neq \emptyset$. It is easy to see that every member of the latter intersection is a choice function of the family $\left\{X_{n}\right.$ : $n \in \mathbb{N}\}$. 
The second assertion follows from the first one and the observation that any finite set $A$ endowed with the discrete metric is a countably compact metric space.

(ii) Fix an infinite set $X$ and let $d$ be the discrete metric on $X$. Clearly, $\mathbf{X}$ is not compact. Hence, by our hypothesis, $\mathbf{X}$ is not countably compact. Thus, $\mathbf{X}$ has a countable open cover $\mathcal{U}$ without a finite subcover, meaning that $\mathcal{U}$ is countably infinite. Hence, IWDI holds true.

In view of Theorem 3, separability is the weakest property $p$ such that every sequentially compact metric space having the property $p$ is compact. In Theorem 14 below we show that the statement "Every complete (resp. Cantor complete) and totally bounded metric space is compact" implies "Every complete (resp. Cantor complete) and totally bounded metric space is separable". Hence, separability is the weakest property for the $\mathbf{Z F}$ validity of the implications $\mathbf{c t b} \rightarrow \mathbf{c}$ ("Every complete and totally bounded metric space is compact") and cctb $\rightarrow$ c ("Every Cantor complete and totally bounded metric space is compact").

In Corollary 13 we observe in $\mathbf{Z F}$ that a quasi separable metric space is compact iff it is limit point compact iff it is countably compact. In view of the latter equivalences one may ask:

- Is there a property $p$ weaker than quasi separability such that the implication lpc $\rightarrow \mathbf{c}$ (resp. $\mathbf{c c} \rightarrow \mathbf{c}$ ) is true in $\mathbf{Z F}$ ?

We show in Theorem 16 that for the limit point compact case the answer is negative, but for the countably compact case the answer is affirmative.

TheOrem 5 ([10]). CAC implies "Every totally bounded metric space is separable".

It is known (see e.g. 44) that in the $\mathbf{Z F}$ model $\mathcal{M} 1$, CUC holds true. By Remark 1 , the subspace $\mathbf{X}, X=(0,1) \cap A$, of the real line $\mathbb{R}$ is totally bounded but not separable. Hence, CUC does not imply the statement "Every totally bounded metric space is separable". So, it seems that the latter statement is strong enough (we will see in Proposition 8 that it implies $\mathbf{C A C}(\mathbb{R})$ ) and one may ask, in view of Theorem 5, if it implies CAC. We show in Theorem 9 that the answer is negative. Since CUC clearly implies "Every strongly totally bounded metric space is separable", we see that strong total boundedness is really stronger than total boundedness in $\mathbf{Z F}$.

3. Almost separability, strong almost separability and strong total boundedness. Clearly, in ZFC the notions of strongly totally bounded, strongly precompact, totally bounded and precompact metric spaces coincide. Likewise, the notions of quasi separable, $\omega$-quasi separable, almost separable, strongly almost separable and separable metric spaces also coincide. 
However, as expected, in $\mathbf{Z F}$ they are no longer equivalent. We demonstrate this fact in propositions to follow.

By Remark 1 , the subspace $\mathbf{X}, X=(0,1) \cap A$, of the real line $\mathbb{R}$ in $\mathcal{M} 1$ is second countable and totally bounded but not separable. Our first result in this section shows in $\mathbf{Z F}$ that second countable and strongly totally bounded metric spaces are separable.

Proposition 6. $(\mathbf{Z F})$ Let $\mathbf{X}=(X, d)$ be a metric space. Then:

(i) $\mathbf{X}$ is separable iff it is second countable and quasi separable.

(ii) If $\mathbf{X}$ is strongly totally bounded then it is quasi separable. The converse fails.

Proof. (i) $(\rightarrow)$ This is straightforward.

$(\leftarrow)$ Fix a countable base $\mathcal{B}=\left\{B_{n}: n \in \mathbb{N}\right\}$ and a sequence $\left(D_{n}\right)_{n \in \mathbb{N}}$ of finite subsets of $X$ such that $D=\bigcup\left\{D_{n}: n \in \mathbb{N}\right\}$ is dense in $\mathbf{X}$. Use the well-ordering of $\mathcal{B}$ to define, for every $n \in \mathbb{N}$, a well-ordering on $D_{n}$. Then $D$ is countable as a countable union of well-ordered finite sets.

(ii) The first assertion of (ii) is obvious. For the second, simply note that the real line is separable but not totally bounded, hence not strongly totally bounded.

\section{PRoposition 7.}

(i) Each of the statements: "Every almost separable metric space is separable" and "Every strongly almost separable metric space is separable" implies CUC. In addition CUC implies the second statement.

(ii) Each of the statements: "Every strongly totally bounded metric space is separable", "Every quasi separable metric space is separable" and "Every strongly precompact metric space is separable" implies $\mathbf{C A C}_{\text {fin }}$.

(iii) Each of the statements: "Every strongly almost separable metric space is quasi separable" and "Every almost separable metric space is quasi separable" implies $\mathbf{C A C}_{\omega}(\mathbb{R})$.

(iv) Each of the statements: "Every quasi separable metric space is strongly almost separable" and "Every quasi separable metric space is almost separable" implies $\mathbf{C A C}_{\mathrm{fin}}$.

(v) Each of the statements: "Every $\omega$-quasi separable metric space is strongly almost separable", "Every $\omega$-quasi separable metric space is separable" and "Every $\omega$-quasi separable metric space is almost separable" is equivalent to $\mathbf{C U C}$.

Proof. (i) Fix a disjoint family $\mathcal{A}=\left\{A_{i}: i \in \mathbb{N}\right\}$ of countable sets. Let $X=\bigcup\left\{A_{i}: i \in \mathbb{N}\right\}$ and $d: X \times X \rightarrow \mathbb{R}$ be the metric given by 


$$
d(x, y)= \begin{cases}0 & \text { if } x=y, \\ 1 / n & \text { if } x \in A_{n}, y \in A_{m} \text { and } n \leq m .\end{cases}
$$

We claim that for every $n \in \mathbb{N}$,

$$
D_{n}=\bigcup\left\{A_{i}: i \leq n+1\right\}
$$

is a countable $1 / n$-dense subset of $\mathbf{X}$. Indeed, for every $n \in \mathbb{N}, D_{n}$ being a finite union of countable sets is countable. Furthermore, if $x \in X \backslash D_{n}$ then $x \in A_{m}$ for some $m>n+1$. Hence, for every $y \in A_{n+1}, 1 / n>1 /(n+1)=$ $d(x, y) \geq d\left(x, D_{n}\right)$, and so $D_{n}$ is $1 / n$-dense in $\mathbf{X}$ as claimed. Hence, $\mathbf{X}$ is strongly almost separable. Fix, by our hypothesis, a countable dense set

$$
Q=\left\{q_{n}: n \in \mathbb{N}\right\}
$$

of $\mathbf{X}$. Since $d$ induces the discrete topology on $X$, it follows that $\mathcal{B}=\{\{x\}$ : $x \in X\}$ is a base for $\mathbf{X}$. Hence, for every $x \in X, x=q_{n}$ for some $n \in \mathbb{N}$, meaning that $X=Q$ is countable as required.

The second assertion is straightforward.

(ii) Fix a disjoint family $\mathcal{A}=\left\{A_{i}: i \in \mathbb{N}\right\}$ of non-empty finite sets. Let $X, d$ and $D_{n}, n \in \mathbb{N}$, be given by (1), (2) and (3) respectively. Clearly, for every $n \in \mathbb{N}, D_{n}$ is a $1 / n$-net of $\mathbf{X}$ and

$$
\mathcal{B}_{n}=\left\{B(x, 1 / n): x \in \bigcup\left\{A_{i}: i \leq n+1\right\}\right\}
$$

is a countable open cover of $\mathbf{X}$ consisting of open balls of radius $1 / n$. Hence, $\mathbf{X}$ is strongly totally bounded, strongly precompact and quasi separable. Hence, in any case, $\mathbf{X}$ has a countable base $\mathcal{B}$. Since $d$ induces the discrete topology, it follows that $\{\{x\}: x \in X\} \subseteq \mathcal{B}$. Hence, $X$ is countable, $\mathcal{A}$ has a choice set and $\mathbf{C A} \mathbf{C}_{\text {fin }}$ holds true.

(iii) Fix a disjoint family $\mathcal{A}=\left\{A_{i}: i \in \mathbb{N}\right\}$ of countable subsets of $\mathbb{R}$ and let $X=\bigcup\left\{A_{i}: i \in \mathbb{N}\right\}$. Without loss of generality we may assume that $A_{n} \subseteq(1 /(n+1), 1 / n)$ for every $n \in \mathbb{N}$. Clearly, for every $n \in \mathbb{N}$, $D_{n}=\bigcup\left\{A_{i}: i \leq n\right\}$ is a countable $1 / n$-dense subspace $\mathbf{X}$ of $\mathbb{R}$, and the open balls of radius $1 / n$ whose centers are from $D_{n}$ cover $\mathbf{X}$. So, $\mathbf{X}$ is almost separable and strongly almost separable. Hence, by our hypothesis, $\mathbf{X}$ is quasi separable. Fix a family $\mathcal{Q}=\left\{Q_{n}: n \in \mathbb{N}\right\}$ of finite subsets of $\mathbf{X}$ with dense union. Since for every $n \in \mathbb{N}, A_{n}$ is an open subset of $\mathbf{X}$ and $Q=\bigcup \mathcal{Q}$ is dense in $\mathbf{X}$, it follows that there exists the least $k_{n}$ such that $Q_{k_{n}} \cap A_{n} \neq \emptyset$. Clearly, the mapping $A_{n} \mapsto \min \left(Q_{k_{n}} \cap A_{n}\right)$ is a choice function for $\mathcal{A}$, finishing the proof of (iii).

(iv), (v) $(\rightarrow)$ Fix a disjoint family $\mathcal{A}=\left\{A_{i}: i \in \mathbb{N}\right\}$ of non-empty finite (resp. countable) sets. Let $X=\bigcup\left\{A_{i}: i \in \mathbb{N}\right\}$ and let $d$ be the discrete metric on $X$. Clearly, $\mathbf{X}$ is quasi separable (resp. $\omega$-quasi separable). By our hypothesis, $\mathbf{X}$ has a $1 / 2$-net (resp. a countable 1/2-dense subset). However, 
the only $1 / 2$-net (resp. countable $1 / 2$-dense subset) subset of $\mathbf{X}$ is $X$ itself. Hence, $X$ is countable and $\mathcal{A}$ has a choice set.

$(\mathrm{v})(\leftarrow)$ This is obvious.

Clearly, every strongly totally bounded (resp. every strongly precompact) metric space is totally bounded (resp. precompact). Next we show that the statements "Every totally bounded metric space is strongly totally bounded" and "Every precompact metric space is strongly precompact" cannot be proved in $\mathbf{Z F}$. In contrast to the well known fact that the properties of total boundedness and precompactness are hereditary in $\mathbf{Z F}$, we also show that strong total boundedness and strong precompactness are not hereditary.

\section{Proposition 8.}

(i) Each of the statements: "Every totally bounded metric space is strongly totally bounded", "Every totally bounded metric space is separable" and "Every precompact metric space is strongly precompact" implies $\mathbf{C A C}(\mathbb{R})$.

(ii) Each of the statements: "Every subspace of a strongly totally bounded metric space is strongly totally bounded" and "Every subspace of a strongly precompact metric space is strongly precompact" implies $\mathrm{CAC}(\mathbb{R})$.

Proof. (i) Fix a family $\mathcal{A}=\left\{A_{n}: n \in \mathbb{N}\right\}$ of dense subsets of $\mathbb{R}$. For every $n \in \mathbb{N}$, let $X_{n}=(1 /(n+1), 1 / n) \cap A_{n}$, and set $X=\bigcup\left\{X_{n}: n \in \mathbb{N}\right\}$. Clearly, the subspace $\mathbf{X}$ of $\mathbb{R}$ is totally bounded. Let, by our hypothesis, $\left(D_{n}\right)_{n \in \mathbb{N}}$, $\left(\mathcal{U}_{n}\right)_{n \in \mathbb{N}}$ satisfy the definition of strongly totally bounded and strongly precompact respectively for the space $\mathbf{X}$, and $S$ be a countable dense subset of X. Clearly the sets $D=\bigcup\left\{D_{n}: n \in \mathbb{N}\right\}$ and $Q=\bigcup\left\{Q_{n}: n \in \mathbb{N}\right\}$, where for every $n \in \mathbb{N}, Q_{n}$ denotes the centers of the balls of $\mathcal{U}_{n}$, are dense in $\mathbf{X}$. Since $\bar{X}=[0,1]$, it follows that for every $n \in \mathbb{N}$ each ball in $\mathcal{U}_{n}$ has a unique center. Hence, for every $n \in \mathbb{N}, Q_{n}$ is a $1 / n$-net of $\mathbf{X}$. Assume for convenience that $Q_{n}=D_{n}$ for every $n \in \mathbb{N}$. It is easy to see that $D$ is countable and we may also assume that $D=S$. Since for every $n \in \mathbb{N}, X_{n}$ is open in $\mathbf{X}$, it follows that $X_{n} \cap D \neq \emptyset$. Clearly the mapping $A_{n} \mapsto d_{n}$, where $d_{n}$ is the first member of $D$ lying in $X_{n}$, is a choice function of $\mathcal{A}$.

(ii) Fix $\mathcal{A}$ and $\mathbf{X}$ as in part (i). We claim that the subspace $[0,1]$ of $\mathbb{R}$ is strongly totally bounded. Indeed, for every $\varepsilon>0, \mathcal{U}_{\varepsilon}=\{(q-\varepsilon, q+\varepsilon): q \in$ $[0,1] \cap \mathbb{Q}\}$ is an open cover of $[0,1]$. Hence, by the compactness of $[0,1]$ it has a finite subcover. Let $Q_{\varepsilon}$ be the first member of $[[0,1] \cap \mathbb{Q}]^{<\omega}$ such that $[0,1] \subseteq \bigcup\left\{(q-\varepsilon, q+\varepsilon): q \in Q_{\varepsilon}\right\}$. Clearly, $Q_{\varepsilon}$ is an $\varepsilon$-net of $[0,1]$. Hence, $[0,1]$ is strongly totally bounded and strongly precompact. Thus, the subspace $\mathbf{X}$ of $[0,1]$ of part (i) has the same properties and $\mathcal{A}$ has a choice function. 
THEOREM 9. The negation of "Every strongly precompact (resp. totally bounded) metric space is separable implies $\mathbf{C A C}$ " is consistent with $\mathbf{Z F}$.

Proof. We claim that in the Jech/Levy/Pincus model $\mathcal{N} 16$ in 4$](\mathcal{N} 16$ is obtained by considering a set $A$ of atoms of cardinality $\aleph_{\omega}$ and letting the ideal of supports be the set of all subsets of $A$ of cardinality less than $\aleph_{\omega}$ ), the statement "Every strongly precompact (resp. totally bounded) metric space is separable" holds true. We recall (see e.g. [4, p. 194] and [5, Theorem 8.9 , p. 124]) that the statement

$W_{\aleph_{\omega}}$ : For every $i \in \omega$, for every set $X$, either $|X| \leq \aleph_{i}$ or $\aleph_{i} \leq|X|$ holds true in $\mathcal{N} 16$. Without loss of generality we may assume that the kernel of $\mathcal{N} 16$ satisfies $|\mathcal{P}(\mathbb{R})|=\aleph_{2}$.

Fix a strongly precompact (resp. totally bounded) metric space $\mathbf{X}=$ $(X, d)$ in $\mathcal{N} 16$. We show that $\mathbf{X}$ is separable. By $W_{\aleph_{\omega}}$, either $X$ is wellorderable or it contains a well-ordered subset $Y$ of size $\aleph_{2}$. We will show that the second case is excluded. We need the following claim.

Claim 1. Y is strongly precompact (resp. totally bounded).

Proof of Claim 1. Since the notions of strong total boundedness, total boundedness and strong precompactness coincide for well-ordered metric spaces, it suffices to show that $\mathbf{Y}$ is totally bounded. Assume the contrary and fix $n \in \mathbb{N}$ such that $\mathbf{Y}$ has no $1 / n$-nets. Via a straightforward induction we can show that $\mathbf{Y}$ has a subspace $\mathbf{A}, A=\left\{a_{i}: i \in \aleph_{0}\right\}$ such that

$$
\text { for all distinct } i, j \in \aleph_{0}, \quad d\left(a_{i}, a_{j}\right) \geq 1 / n \text {. }
$$

Since $\mathbf{X}$ is totally bounded, for $\varepsilon=1 /(3 n)$ there exists an $\varepsilon$-net $C=\left\{c_{i}\right.$ : $i \leq k\}$ of $\mathbf{X}$, i.e., $X=\bigcup\left\{B\left(c_{i}, 1 /(3 n)\right): i \leq k\right\}$. It is easy to see that there exist $i \leq k$ and $x, y \in A \cap B\left(c_{i}, 1 /(3 n)\right)$ with $x \neq y$. Hence

$$
1 / n \leq d(x, y) \leq d\left(x, c_{i}\right)+d\left(c_{i}, y\right)<2 /(3 n) .
$$

This is a contradiction. So, $\mathbf{Y}$ is totally bounded as required.

Use Claim 1 and the well-ordering of $Y$ to define for every $n \in \mathbb{N}$ a $1 / n$-net $D_{n}$ of $\mathbf{Y}$. Then $D=\bigcup\left\{D_{n}: n \in \mathbb{N}\right\}$ is a countable, dense subset of $\mathbf{Y}$. We have

$$
\aleph_{2}=|Y| \leq|\mathcal{P}(D)|=\aleph_{1}
$$

This is a contradiction. Hence, $\mathbf{X}$ does not have subsets of size $\aleph_{2}$, and consequently $|X| \leq \aleph_{1}$. Working as in Claim 1 with $\mathbf{X}$ in place of $\mathbf{Y}$, we can show that $\mathbf{X}$ is separable. Hence, "Every strongly precompact (resp. totally bounded) metric space is separable" holds true in $\mathcal{N} 16$.

It is known (see e.g. [4, p. 194] and [5, Theorem 8.9, p. 124]) that CAC fails in $\mathcal{N} 16$. Since $W_{\aleph_{\omega}}$ and $\mathbf{C A C}$ are transferable in $\mathbf{Z F}$, it follows that 
there exists a ZF model satisfying "Every strongly precompact (resp. totally bounded) metric space is separable" and the negation of $\mathbf{C A C}$, finishing the proof of the theorem.

It is well known (see e.g. [1]) that $\mathbf{C A C}(\mathbb{R})$ is equivalent to the assertion "Every second countable metric space $\mathbf{X}=(X, d)$ is separable". We show next that in the latter equivalence separability can be replaced by almost separability.

THEOREM 10. The statement "Every second countable metric space $\mathbf{X}=$ $(X, d)$ is almost separable" is equivalent to $\mathbf{C A C}(\mathbb{R})$.

Proof. $(\leftarrow)$ This is straightforward.

$(\rightarrow)$ Fix a disjoint family $\mathcal{A}=\left\{A_{n}: n \in \mathbb{N}\right\}$ of non-empty dense subsets of $\mathbb{R}$. Since for any $x, y \in \mathbb{R}$ with $x<y$ a homeomorphism $f: \mathbb{R} \rightarrow(x, y)$ can be defined in $\mathbf{Z F}$, we may assume that for all $n \in \mathbb{N}, A_{n} \subseteq(2 n, 2(n+1))$ is dense in the subspace $(2 n, 2(n+1))$ of $\mathbb{R}$. Let $X=\bigcup\left\{A_{n}: n \in \mathbb{N}\right\}$ and $d: X \times X \rightarrow \mathbb{R}$ be the restriction of the usual metric of $\mathbb{R}$ to $X$. Since $\mathbb{R}$ is second countable, so is $\mathbf{X}$. Fix, by our hypothesis, a countable $1 / 2$-dense subset $D=\left\{d_{n}: n \in \mathbb{N}\right\}$ of $\mathbf{X}$. Hence for every $n \in \mathbb{N}, A_{n}$ is an open subset of $\mathbf{X}$ such that $A_{n} \cap D \neq \emptyset$. [Indeed, for every $x \in A_{n}$ there exists $n_{x} \in \mathbb{N}$ such that $x \in B\left(d_{n_{x}}, 1 / 2\right)$. Fix $x \in A_{n}$ such that $d(x, 2 n+1)<1 / 3$; then $x \in$ $B\left(d_{n_{x}}, 1 / 2\right)$, hence $d\left(d_{n_{x}}, 2 n+1\right) \leq d\left(d_{n_{x}}, x\right)+d(x, 2 n+1)<1 / 2+1 / 3<1$, meaning that $d_{n_{x}} \in A_{n} \cap D$.] Clearly, $c=\left\{c_{n}: n \in \mathbb{N}\right\}$, where for every $n \in \mathbb{N}, c_{n}$ is the first member of $D$ which is in $A_{n}$, is a choice set for $\mathcal{A}$, finishing the proof of the theorem.

4. Sequential, limit point and countable compactness of metric spaces. In the first result of this section we show that, in contrast to Theorem 1, none of the statements: "A sequentially compact, quasi separable metric space is compact" and "A complete, totally bounded, quasi separable metric space is compact" is provable in $\mathbf{Z F}$.

THEOREM 11. Each of the statements:

(i) "A sequentially compact, quasi separable metric space is compact",

(ii) "A complete, totally bounded, quasi separable metric space is compact",

(iii) "A limit point compact (resp. countably compact) quasi separable metric space is separable"

implies $\mathbf{C A C}_{\text {fin }}$.

Proof. (i)\&(ii) Assume the contrary and fix a disjoint family $\mathcal{A}=$ $\left\{A_{i}: i \in \mathbb{N}\right\}$ of finite, non-empty sets without a partial choice set. Set $X=\bigcup\left\{A_{i}: i \in \mathbb{N}\right\}$ and let $d: X \times X \rightarrow \mathbb{R}$ be the metric given by (1). 
It is easy to see that $\mathbf{X}$ is quasi separable and totally bounded. Since every sequence in $\mathbf{X}$ has a finite range, it follows that $\mathbf{X}$ is complete and sequentially compact. Hence, by our hypothesis, $\mathbf{X}$ is compact. However, the open cover $\mathcal{U}=\{\{x\}: x \in X\}$ of $\mathbf{X}$ has no finite subcover, a contradiction. Thus, $\mathbf{C A} \mathbf{C}_{\text {fin }}$ holds true as required.

(iii) Fix a disjoint family $\mathcal{A}=\left\{A_{i}: i \in \mathbb{N}\right\}$ of finite non-empty sets. Let $X=\bigcup\left\{A_{i}: i \in \omega\right\} \cup\{\infty\}$ with $\infty \notin \bigcup\left\{A_{i}: i \in \omega\right\}$ and $d: X \times X \rightarrow \mathbb{R}$ be the metric given by

$$
d(x, y)= \begin{cases}0 & \text { if } x=y \\ 1 / n & \text { if } x \in A_{n}, y \in A_{m} \text { and } n \leq m \\ 1 / n & \text { if } x \in A_{n}, y=\infty\end{cases}
$$

Clearly, $\mathbf{X}$ is quasi separable and compact. Thus, $\mathbf{X}$ is limit point compact (resp. countably compact) and, by our hypothesis, separable. It is not hard now to define a choice set for $\mathcal{A}$.

In view of Theorem 11 and the fact that a quasi separable pseudometric space $\mathbf{X}=(X, d)$ is compact iff it is limit point compact (see e.g. [8]), one may suspect that "A limit point compact, quasi separable metric space is compact" is true. We show next that this is indeed the case.

Theorem 12.

(i) $(\mathbf{Z F})$ Let $\mathbf{X}=(X, d)$ be a quasi separable metric space. Then $\mathbf{X}$ is compact iff it is limit point compact.

(ii) $(\mathbf{Z F})$ Let $\mathbf{X}=(X, d)$ be an $\omega$-quasi separable metric space. Then $\mathbf{X}$ is compact iff it is countably compact.

Proof. (i) $(\rightarrow)$ This is straightforward.

$(\leftarrow)$ Fix $\mathbf{X}$ as in the statement of the theorem and let $D=\bigcup\left\{D_{n}: n \in \mathbb{N}\right\}$ be a dense subset of $\mathbf{X}$ where $D_{n}$ is finite for every $n \in \mathbb{N}$. We will show that $\mathbf{X}$ is compact. To this end, in view of Theorems 1 and 2 , it suffices to show that $\mathbf{D}$ is totally bounded and $\mathbf{X}$ is Lebesgue.

To prove that $\mathbf{D}$ is totally bounded, assume, aiming for a contradiction, that there exists some $\varepsilon>0$ such that $D$ cannot be covered by finitely many open discs of radius $\varepsilon$. Via a straightforward induction we construct a family $Q=\left\{\emptyset \neq Q_{k_{n}} \subseteq D_{k_{n}}: n \in \mathbb{N}\right\}$ such that $\left(k_{n}\right)_{n \in \mathbb{N}}$ is a strictly increasing sequence of natural numbers and for all $n \in \mathbb{N}$,

$$
\bigcup\left\{B(x, \varepsilon): x \in \bigcup\left\{Q_{k_{i}}: i \in n\right\}\right\} \cap Q_{k_{n}}=\emptyset,
$$

i.e., $d\left(Q_{k_{n}}, Q_{k_{m}}\right) \geq \varepsilon$ for all distinct $n, m \in \mathbb{N}$. We begin the induction by setting $k_{1}=1$ and $Q_{k_{1}}=D_{k_{1}}$. Assume that $k_{1}<\cdots<k_{n}$ have been 
constructed subject to (4). By our hypothesis, $W_{n}=\bigcup\{B(x, \varepsilon): x \in$ $\left.\bigcup\left\{Q_{k_{i}}: i \leq n\right\}\right\} \neq D$. Let

$$
k_{n+1}=\min \left\{i \in \mathbb{N}: i>k_{n}, D_{i} \backslash W_{n} \neq \emptyset\right\}
$$

and set $Q_{k_{n+1}}=D_{k_{n+1}} \backslash W_{n}$, terminating the induction.

Since $S=\bigcup Q$ is infinite, it follows by our hypothesis that $S$ has a limit point $s$. Hence, $B(s, \varepsilon / 2)$ meets $S$ in an infinite set. Since each $Q_{k_{n}}, n \in \mathbb{N}$, is finite, there exist distinct $n, m \in \mathbb{N}$ with

$$
Q_{k_{n}} \cap B(s, \varepsilon / 2) \neq \emptyset \quad \text { and } \quad Q_{k_{m}} \cap B(s, \varepsilon / 2) \neq \emptyset .
$$

Fix $x_{n}$ and $x_{m}$ in the last two intersections respectively. It follows that $\varepsilon \leq d\left(x_{n}, x_{m}\right) \leq d\left(x_{n}, s\right)+d\left(s, x_{m}\right)<\varepsilon$. This contradiction shows that $\mathbf{X}$ is totally bounded as claimed.

To see that $\mathbf{X}$ is Lebesgue, it suffices in view of Theorem 2 to show that for every open cover $\mathcal{U}$ of $\mathbf{X}$ there exists a $\delta>0$ such that for all $d \in D$ there are $U \in \mathcal{U}$ with $B(d, \delta) \subseteq U$. Assume, aiming for a contradiction, that $\mathcal{U}$ is an open cover of $\mathbf{X}$ such that for every $m \in \mathbb{N}$, there are $n \in \mathbb{N}$ and $d \in D_{n}$ with $B(d, 1 / m) \nsubseteq U$ for all $U \in \mathcal{U}$. For every $m \in \mathbb{N}$, let $n_{m}$ be the least $n \in \mathbb{N}$ such that for some $d \in D_{n}, B(d, 1 / m) \nsubseteq \subseteq U$ for all $U \in \mathcal{U}$. Set

$$
K_{m}=\left\{d \in D_{n_{m}}: B(d, 1 / m) \nsubseteq \mathbb{U} \text { for all } U \in \mathcal{U}\right\}
$$

and let $K=\bigcup\left\{K_{m}: m \in \mathbb{N}\right\}$. We consider the following two cases:

(1) $K$ is finite. In this case, there exists $d \in K$ such that $d \in K_{m}$ for infinitely many $m \in \mathbb{N}$. Since $\mathcal{U}$ is an open cover of $\mathbf{X}$, there is $U \in \mathcal{U}$ with $d \in U$. Since $U$ is open, there exists $m_{0} \in \mathbb{N}$ such that $B\left(d, 1 / m_{0}\right) \subseteq U$. Fix $m \geq m_{0}$ such that $d \in K_{m}$. Then $B(d, 1 / m) \subseteq B\left(d, 1 / m_{0}\right) \subseteq U$. This is a contradiction showing that (1) cannot be the case.

(2) $K$ is infinite. In this case, we use the fact that $\mathbf{X}$ is limit point compact and fix a limit point $t$ of $K$. Since each $K_{m}, m \in \mathbb{N}$, is finite, every open ball centered at $t$ meets non-trivially infinitely many $K_{m}$ 's. As in case (1) there exist $U \in \mathcal{U}$ and $m_{0} \in \mathbb{N}$ such that $B\left(t, 1 / m_{0}\right) \subseteq U$. Fix $x \in K_{m} \cap B\left(t, 1 /\left(3 m_{0}\right)\right)$ for some $m \geq 3 m_{0}$. Then, $B(x, 1 / m) \subseteq B\left(t, 1 / m_{0}\right)$. Indeed, if $y \in B(x, 1 / m)$ then

$$
d(t, y) \leq d(t, x)+d(x, y)<1 /\left(3 m_{0}\right)+1 / m \leq 2 /\left(3 m_{0}\right)<1 / m_{0} .
$$

Since $B\left(t, 1 / m_{0}\right) \subseteq U$, we see that $B(x, 1 / m) \subseteq U$ and $x \in K_{m}$. This leads to a contradiction. So, case (2) cannot occur either, finishing the proof of (i).

(ii) $(\rightarrow)$ This is obvious.

$(\leftarrow)$ Fix an $\omega$-quasi separable, countably compact metric space $\mathbf{X}$ and let $D=\bigcup\left\{D_{n}: n \in \mathbb{N}\right\}$ be a dense subset of $X$ such that $D_{n}$ is countable for every $n \in \mathbb{N}$. Clearly,

$$
\mathcal{B}=\left\{B(x, 1 / m): x \in D_{n}, n, m \in \mathbb{N}\right\}
$$


is a base for $\mathbf{X}$. We show that every cover $\mathcal{U} \subseteq \mathcal{B}$ of $\mathbf{X}$ has a finite subcover. Fix such an open cover of $\mathbf{X}$. For every $n \in \mathbb{N}$, let

$$
O_{n}=\bigcup\left\{B \in \mathcal{U}: B \text { has a center in } D_{n}\right\} .
$$

Clearly, $\mathcal{W}=\left\{O_{n}: n \in \mathbb{N}\right\}$ is a countable open cover of $\mathbf{X}$. Hence, by our hypothesis, $\mathcal{W}$ has a finite subcover $\mathcal{V}=\left\{O_{n_{i}}: i \leq k\right\}$. Since

$$
\mathcal{Q}=\left\{B \in \mathcal{U}: B \text { has a center in } D_{n_{i}}, i \leq k\right\}
$$

is clearly a countable subcover of $\mathcal{U}$, it follows by the countable compactness of $\mathbf{X}$ that $\mathcal{Q}$ has a finite subcover $\mathcal{G}$. Clearly, $\mathcal{G}$ is a subcover of $\mathcal{U}$, and $\mathbf{X}$ is compact as required.

Corollary 13. (ZF) Let $\mathbf{X}=(X, d)$ be a quasi separable metric space. Then the following are equivalent:

(i) $\mathbf{X}$ is compact.

(ii) $\mathbf{X}$ is countably compact.

(iii) $\mathbf{X}$ is limit point compact.

THEOREM 14. The statement "Every complete (resp. Cantor complete) and totally bounded metric space is compact" implies each of the following:

(i) CPCMC: If $\left\{\mathbf{X}_{n}=\left(X_{n}, d_{n}\right): n \in \mathbb{N}\right\}$ is a family of compact metric spaces then $\mathbf{X}=\prod_{n \in \mathbb{N}} \mathbf{X}_{n}$ is compact.

(ii) $\mathbf{C A C}_{\mathrm{CM}}$ : For every metric space $\mathbf{X}=(X, d)$, every family $\mathcal{A}=\left\{A_{i}\right.$ : $i \in \omega\}$ of non-empty compact subsets of $\mathbf{X}$ has a choice function.

(iii) Every complete (resp. Cantor complete) and totally bounded metric space is separable.

Proof. (i) Fix a family $\left\{\mathbf{X}_{n}=\left(X_{n}, d_{n}\right): n \in \mathbb{N}\right\}$ of compact metric spaces and let $\mathbf{X}=\prod_{n \in \mathbb{N}} \mathbf{X}_{n}$. Since a compact metric space is complete (resp. Cantor complete) and totally bounded, Theorem 2 implies that $\mathbf{X}$ is complete (resp. Cantor complete) and totally bounded. Hence, by our hypothesis, $\mathbf{X}$ is compact as required.

(ii)\&(iii) can be proved as in the proof of Theorem 3(ii)\&(iii) respectively.

As a corollary to Theorem 14 we get:

COROLlary 15. (ZF) There is no property $p$ strictly weaker than separability such that the statement "Every complete (resp. Cantor complete) and totally bounded metric space having the property $p$ is compact" is true in $\mathbf{Z F}$.

The following remark was suggested by the referee.

REMARK 2. Theorem 14 states that if every complete and totally bounded metric space is compact, then also every complete and totally bounded metric space is separable. This is also our definition of being the 
weakest property under which a complete and totally bounded metric space having this property is compact. In order to make a distinction between possible other definitions we will say that a property is I-weakest if it is weakest in the sense of Theorem 14. On the other hand, we can consider another natural definition. We say that property $p$ is $I I$-weakest if:

- under the property $p$ every complete and totally bounded metric space is compact,

- if $q$ is a property under which every complete and totally bounded metric space is compact, then every complete and totally bounded metric space with the property $q$ also has the property $p$.

If a property is II-weakest, then it is also I-weakest, but the converse is not true: separability is I-weakest, but if it were also II-weakest, then every compact metric space would be separable.

THEOREM 16. There is no property $p$ strictly weaker than quasi separability such that the statement "Every limit point compact metric space having the property $p$ is compact" is true in $\mathbf{Z F}$.

Proof. First we establish the following claim:

Claim 1. The statement "Every limit point compact metric space is compact" implies $\mathbf{C M C}_{\mathrm{MC}}$ ("For every metric space $\mathbf{X}=(X, d)$, every disjoint family $\mathcal{A}=\left\{A_{i}: i \in \omega\right\}$ of non-empty compact subsets of $\mathbf{X}$ has a multiple choice function, i.e., a function $f: \mathcal{A} \rightarrow \bigcup \mathcal{A}$ such that for every $A \in \mathcal{A}$, $f(A)$ is a non-empty finite subset of $A$ ").

Proof of Claim 1. Fix a metric space $\mathbf{X}=(X, d)$ and let $\mathcal{A}=\left\{A_{i}: i \in \mathbb{N}\right\}$ be a disjoint family of non-empty compact subsets of $\mathbf{X}$. Without loss of generality we may assume that the diameter of $X$ is $\leq 1$. Clearly for every $n \in \mathbb{N}$ the product $\mathbf{Y}_{n}=\prod_{i \leq n} \mathbf{A}_{i}$ is compact. It is easy to verify that any partial multiple choice function $f$ of the family $\left\{Y_{n}: n \in \mathbb{N}\right\}$ (there is an infinite $\mathbb{N}_{f} \subseteq \mathbb{N}$ such that for every $n \in \mathbb{N}_{f}, f(n)$ is a finite non-empty subset of $Y_{n}$ ) leads to a multiple choice set of $\mathcal{A}$. So, we assume that $\mathcal{A}$ has no partial multiple choice function. Let $Y=\bigcup \mathcal{A}$ and $\sigma: Y \times Y \rightarrow \mathbb{R}$ be the metric given by the rule

$$
\sigma(x, y)= \begin{cases}d(x, y) & \text { if } x, y \in A_{i} \\ 1 & \text { otherwise }\end{cases}
$$

Since $\mathcal{A}$ has no partial multiple choice function, every infinite subset $K$ of $Y$ meets in a finite non-empty set only finitely many members of $\mathcal{A}$. Hence, $K \cap A_{i}$ is an infinite subset of $A_{i}$ for some $i \in \mathbb{N}$. Since $A_{i}$ is compact, $K \cap A_{i}$ has a limit point $x \in A_{i}$. Clearly, $x$ is a limit point of $K$ in $\mathbf{Y}$, and consequently, by our hypothesis, $\mathbf{Y}$ is compact. This leads to a contradiction 
$\left(\mathcal{U}=\left\{A_{i}: i \in \mathbb{N}\right\}\right.$ is an open cover of $\mathbf{Y}$ without a finite subcover $)$. Hence, $\mathbf{C M C}_{\mathrm{MC}}$ holds true as claimed.

We continue with the proof of the theorem by assuming the contrary and letting $p$ be a property strictly weaker than quasi separability such that every limit point compact metric space having the property $p$ is compact. Fix a limit point compact, non-quasi separable metric space $\mathbf{X}=(X, d)$ having the property $p$. We will show that $\mathbf{X}$ is quasi separable and thus arrive at a contradiction. By our hypothesis, $\mathbf{X}$ is compact, and consequently totally bounded. Hence, for every $\varepsilon>0$ there exist $x_{i} \in X, i=1, \ldots, n$, such that $\bigcup\left\{\overline{B\left(x_{i}, \varepsilon\right)}: i \in n\right\}=X$. For every $k \in \mathbb{N}$, let $m_{k}$ denote the least natural number $m$ for which there exists a set $\left\{x_{i}: i \in m\right\} \subseteq X$ with $\bigcup\left\{\overline{B\left(x_{i}, 1 / k\right)}: i \in m\right\}=X$, and set $\mathbf{Y}_{k}=\left(X^{m_{k}}, \rho_{k}\right)$, where $\rho_{k}$ is the uniform metric on $X^{m_{k}}$. Since $\mathbf{X}$ is compact, so is $\mathbf{Y}_{k}$. Working as in the proof of Theorem 3 we can show that for every $k \in \mathbb{N}$, the set

$$
G_{k}=\left\{f \in X^{m_{k}}: \bigcup\left\{\overline{B(f(i), 1 / k)}: i \in m_{k}\right\}=X\right\}
$$

is a (non-empty) closed subset of $\mathbf{Y}_{k}$. Since $\mathbf{Y}_{k}$ is compact, so is $G_{k}$. Thus, by $\mathbf{C M C}_{\mathrm{CM}}$, the disjoint family $\mathcal{G}=\left\{G_{k}: k \in \mathbb{N}\right\}$ of compact sets has a multiple choice function $h$. Clearly, for every $k \in \mathbb{N} \backslash\{1\}$,

$$
D_{k}=\left\{f(i): i \in m_{k}, f \in h(k)\right\}
$$

is a $1 /(k-1)$-net of $\mathbf{X}$. Hence, $\mathbf{X}$ is strongly totally bounded, and consequently quasi separable as required, finishing the proof of the theorem.

Acknowledgements. I would like to thank very much the referee for Remark 2 and all his useful comments which improved our paper.

\section{References}

[1] C. Good and I. J. Tree, Continuing horrors of topology without choice, Topology Appl. 63 (1995), 79-90.

[2] G. Gutierres, Total boundedness and the axiom of choice, Appl. Categor. Structures 24 (2016), 457-469.

[3] P. Howard, K. Keremedis, J. E. Rubin, A. Stanley and E. Tachtsis, Non-constructive properties of the real line, Math. Logic Quart. 47 (2001), 423-431.

[4] P. Howard and J. E. Rubin, Consequences of the Axiom of Choice, Math. Surveys Monogr. 59, Amer. Math. Soc., Providence, RI, 1998.

[5] T. Jech, The Axiom of Choice, North-Holland, 1973.

[6] K. Keremedis, On the relative strength of forms of compactness of metric spaces and their countable productivity in ZF, Topology Appl. 159 (2012), 3396-3403.

[7] K. Keremedis, On sequentially compact and related notions of compactness of metric spaces in ZF, Bull. Polish Acad. Sci. Math. 64 (2016), 29-46.

[8] K. Keremedis, On Weierstrass compact pseudometric spaces and a weak form of the axiom of choice, Topology Appl. 108 (2000), 75-78. 
[9] J. R. Munkres, Topology, Prentice-Hall, Englewood Cliffs, NJ, 1975.

[10] J. Nagata, Modern General Topology, North-Holland, 1985.

Kyriakos Keremedis

Department of Mathematics

University of the Aegean

Karlovassi, Samos 83200, Greece

E-mail: kker@aegean.gr 